\title{
Simulation du réacteur de synthèse de méthanol d'Arzew
}

\author{
A. Nacer ${ }^{1}$ et S. Bouroudi ${ }^{1}$ \\ 1 Institut algérien du pétrole, Corporate University - Laboratoire d'Ingénierie et de Simulation des Procédés, \\ avenue du jer Novembre, 35000 Boumerdès - Algérie \\ e-mail : nacer@iap-cu.dz - bouroudi@iap-cu.dz
}

Résumé - Ce travail consiste en une modélisation et une simulation d'un réacteur catalytique étagé et à lits fixes, réalisées à l'aide d'un modèle hétérogène unidimensionnel et à l'état stationnaire, qui tient compte à la fois des gradients de température et de concentrations à la surface et à l'intérieur de la particule de catalyseur, siège de plusieurs réactions chimiques obéissant à un modèle cinétique complexe. L'approche utilisée dans la résolution du modèle consiste en une combinaison de la méthode de Runge-Kutta pour intégrer les équations de la phase fluide et de la collocation orthogonale pour intégrer les équations de diffusion. Le modèle est utilisé pour la simulation du réacteur industriel de synthèse de méthanol d'Arzew. Cette étude a permis de confirmer que la modélisation et la simulation ont une importance majeure dans l'analyse et dans la compréhension du comportement du système réactionnel et en particulier celui de la réaction du gaz à l'eau (Water Gas Shift) et de son facteur d'efficacité. Nous avons montré que la production du méthanol dépend fortement du rapport des pressions partielles $\mathrm{CO}_{2} / \mathrm{CO}$ du gaz de synthèse alimentant le réacteur industriel d'Arzew, l'optimum de ce rapport est de l'ordre de 0,3. La conversion augmente avec l'augmentation de la teneur totale en carbone $\left(\mathrm{CO}_{2}+\mathrm{CO}\right)$ et est maximale pour la même valeur du rapport $\mathrm{CO}_{2} / \mathrm{CO}$ sus-citée.

\begin{abstract}
Simulation of the Methanol Synthesis Reactor at Arzew - The fixed bed reactor is modelled using a steady state heterogeneous mathematical model which takes into account the coupling of chemical reactions with complex kinetics and diffusion resistance. The approach followed to solve the model combines the Runge-Kutta method for the integration of the bulk phase equations, and the orthogonal collocation method for the diffusion equations. This model is applied to simulating a multi fixed bed quench type of methanol synthesis reactor at Arzew. The simulation results emphasis the importance of using a rigorous model to describe the performance of this reactor in analysing and understanding the behaviour of the system such as: the understanding of the behaviour of the water gas shift reaction and the investigation of its effectiveness factor. This work clearly shows that methanol conversion depends mainly on the partial pressure ratio of $\mathrm{CO}_{2} / \mathrm{CO}$ in the synthesis gas feeding the industrial reactor at Arzew. The conversion increases with increasing the total carbon content $\left(\mathrm{CO}_{2}+\mathrm{CO}\right)$ and reaches a maximum when the ratio of $\mathrm{CO}_{2} / \mathrm{CO}$ is equal to 0.3.
\end{abstract}




\section{NOMENCLATURE}

$a_{v} \quad$ surface externe de particules par unité de volume de lit

$C_{i} \quad$ concentration molaire du constituant $i$

$C_{i s}{ }^{s} \quad$ concentration molaire à la surface du catalyseur

$C_{i s} \quad$ concentration molaire à l'intérieur de la particule du catalyseur

$C_{p} \quad$ chaleur spécifique à pression constante

$D_{e i} \quad$ diffusivité effective du constituant $i$

$D_{i j} \quad$ coefficient de diffusion binaire

$D_{K} \quad$ coefficient de diffusion de Knudsen

$d_{p} \quad$ diamètre de la particule du catalyseur

$d_{t} \quad$ diamètre du réacteur

$d z \quad$ pas d'intégration axial

$f \quad$ facteur de frottement

$G \quad$ débit massique du fluide

$h \quad$ coefficient de transfert de chaleur fluide - solide

$K_{j}^{*} \quad$ constante d'équilibre de la réaction $j$

$k_{g i} \quad$ coefficient de transfert de matière externe du constituant $i$

$\mathrm{Nc}, \mathrm{Nr}$ nombre de constituant et de réaction chimique

$P \quad$ pression totale

Re nombre de Reynolds

$R_{j} \quad$ vitesse de la réaction $n^{\circ} j$

$R_{b j} \quad$ vitesse de la réaction $\mathrm{n}^{\circ} j$ au sein du fluide

$T$ température

$T_{\text {ext }} \quad$ température à l'extérieur de tube

$T_{s}^{s}, T_{s} \quad$ température à la surface du catalyseur et à l'intérieur de la particule

$u \quad$ vitesse superficielle du fluide calculée à chaque pas d'intégration (débit sur la section de passage)

$U \quad$ coefficient de transfert de chaleur global

$w_{k} \quad$ valeur de la quadrature à la position $(k)$

$z \quad$ longueur du lit catalytique

\section{Caractères grecs}

$\varepsilon, \varepsilon_{p} \quad$ porosité du lit et de la particule

$\eta_{j} \quad$ facteur d'efficacité de la réaction $j$

$\lambda_{e} \quad$ conductivité thermique effective de la particule

$v_{i j} \quad$ coefficient stœchiométrique du constituant $i$ dans la réaction $j$

$\rho_{g}, \rho_{p}, \rho_{b}$ densité du fluide, de la particule et de la particule au sein du fluide

$\Delta H_{j} \quad$ chaleur de la réaction $n^{\circ} j(\mathrm{kcal} / \mathrm{kmol})$

\section{INTRODUCTION}

En génie chimique, la modélisation des opérations unitaires s'articule sur trois types d'équations, que sont les équations de conservation de masse, d'énergie et de quantité de mouvement $[1,2]$. La performance d'un système catalytique hétérogène dépend en grande partie des caractéristiques du solide, dont l'élément important sur lequel seront appliquées les équations de conservation est la particule du catalyseur et son milieu adjacent. Les équations sont appliquées sur les deux phases fluide et solide. Ce travail consiste en une simulation d'un réacteur étagé à lits fixes adiabatiques utilisant un modèle mathématique résolu avec des méthodes numériques tout en incorporant des routines pour générer les propriétés physico-chimiques ainsi qu'un modèle cinétique.

\section{MODÉLISATION DU RÉACTEUR DE SYNTHÈSE DE MÉTHANOL}

La classification la plus acceptée des modèles de réacteur à lit fixe a été proposée par Froment [3-6]. Dans celle-ci, les modèles sont groupés en deux grandes catégories que sont les modèles pseudo-homogènes et les modèles hétérogènes. Le système réactionnel de synthèse de méthanol est hétérogène et les réactions sont considérées rapides et ayant un effet thermique important. Donc, il est nécessaire de distinguer entre les conditions du fluide, sur la surface et à l'intérieur de la particule du catalyseur. La catégorie du modèle pour la synthèse du méthanol est classée parmi la famille des modèles hétérogènes à gradient interne et externe.

Le modèle mathématique à l'état stationnaire et négligeant la dispersion axiale est donné par les équations suivantes pour un système réactionnel de $N c$ constituants et de $N r$ réactions $[3,4,7]$.

\section{Phase fluide}

L'équation de conservation de masse [3] :

$$
\frac{d(u C)_{i}}{d z}=k_{g i} a_{V}\left(C_{i}-C_{i S}^{S}\right)
$$

L'équation de conservation d'énergie pour un fonctionnement adiabatique [3] :

$$
u \rho_{g} C_{p} \frac{d T}{d z}=h a_{V}\left(T_{S}^{S}-T\right)
$$

L'équation de quantité de mouvement s'écrit :

$$
\frac{d P_{t}}{d z}=-\mathrm{f} \frac{\rho_{\mathrm{g}} u^{2}}{d_{p}}
$$


où $f$ est un facteur de frottement qui est calculé à partir de corrélations, ces dernières pouvant se mettre sous la forme suivante $[3,4]$ :

$$
f=\frac{1-\varepsilon}{\varepsilon^{2}}\left(a+b \frac{1-\varepsilon}{\operatorname{Re}}\right)
$$

\section{Phase solide}

L'équation de conservation de masse [3] :

$$
\frac{D_{e i}}{r^{2}} \frac{d}{d r}\left(r^{2} \frac{d C_{i}}{d r}\right)=-\rho_{P} \sum_{j=1}^{N r} v_{i j} R_{j}
$$

et l'équation de conservation d'énergie :

$$
\frac{\lambda_{e}}{r^{2}} \frac{d}{d r}\left(r^{2} \frac{d T}{d r}\right)=-\rho_{P} \sum_{j=1}^{N r} R_{j}\left(-\Delta H_{j}\right)
$$

Les conditions aux limites sont :

- pour la phase fluide :

$$
C_{i}=C_{i 0}, T=T_{0}, P=P_{0} \text { à } z=0
$$

- pour le solide :

$$
\frac{d C_{i}}{d r}=\frac{d T_{S}}{d r}=0 \quad \text { à } r=0 \text { au centre de la particule }
$$

et à la surface de la particule de catalyseur :

$$
\begin{array}{ll}
k_{g i}\left(C_{i S}^{S}-C_{i}\right)=-D_{e i}\left(\frac{d C_{i}}{d r}\right) & \grave{a} r=\frac{d_{P}}{2} \\
h\left(T_{S}^{S}-T\right)=-\lambda_{e}\left(\frac{d T_{S}}{d r}\right) & \grave{a} r=\frac{d_{P}}{2}
\end{array}
$$

Suite à la complexité de la géométrie des pores du catalyseur, la modélisation acceptable de ce système nécessite l'utilisation d'un modèle simplifié des propriétés physico-chimiques de la particule du catalyseur. Ceci est réalisé en considérant que le solide est un corps pseudohomogène où la structure poreuse est considérée globalement et les transferts de matière et de chaleur sont décrits par un coefficient apparent ou effectif $D_{\mathrm{e}}[8,9]$. Le coefficient de diffusion est donné par la relation suivante :

$$
D_{e}=\frac{\varepsilon_{p} D}{\tau}
$$

$\tau$ est la tortuosité qui varie de 2 à 10 ;

$\varepsilon_{p}$ est la fraction de vide de la particule.
Lorsque les pores sont relativement larges et la pression est élevée, le coefficient de diffusion $(D)$ sera équivalent à celui de la diffusion en phase homogène $\left(D_{\text {im }}\right)$. Il peut être estimé à partir des corrélations relatives à la diffusion moléculaire [10-11].

L'évaluation de la diffusion à l'intérieur de la particule de catalyseur est exprimée souvent par le calcul du facteur d'efficacité de diffusion. Il est défini comme le rapport de la vitesse de réaction à l'intérieur de la particule avec les limitations de diffusion à la vitesse de réaction intrinsèque. Pour un système réactionnel de $\mathrm{Nr}$ réactions, nous utilisons la notion du facteur d'efficacité pour chaque réaction $\left(\eta_{j}\right)$, ce facteur revêt la forme mathématique suivante :

$$
\eta_{j}=\frac{\int R_{j} \rho_{P}\left(\frac{d V}{V_{P}}\right)}{R_{b j} \rho_{p}}
$$

où l'intégration est sur le volume $V_{p}$ de la particule, $V$ est le volume élémentaire considéré, $R_{j}$ est la vitesse à l'intérieur de la particule et $R_{b j}$ est la vitesse au sein du fluide pour la réaction $j$.

De cette équation (10), la vitesse de la réaction globale à l'intérieur de la particule peut être obtenue en multipliant la vitesse calculée aux conditions de la phase fluide par son facteur d'efficacité. En utilisant la vitesse corrigée dans les bilans de la phase fluide, les équations de conservation sont données ci-dessous en utilisant le facteur d'efficacité :

$$
\begin{gathered}
\frac{d\left(u C_{i}\right)}{d z}=\sum_{j=1}^{N r} \eta_{j} v_{i j} R_{b j} \rho_{b} \\
u \rho_{g} C_{p} \frac{d T}{d z}=\sum_{j=1}^{N r} \eta_{j} R_{b j}\left(-\Delta H_{j}\right) \rho_{b}
\end{gathered}
$$

Le modèle cinétique choisi est celui de Froment et al. [12]. Ce modèle est basé sur la conversion du gaz de synthèse sur un catalyseur à base de $\mathrm{Cu} / \mathrm{ZnO} / \mathrm{Al}_{2} \mathrm{O}_{3}$ et tient compte de l'hydrogénation du $\mathrm{CO}_{2}$ pour l'obtention du méthanol ainsi que la réaction gaz à l'eau (Water Gas Shift) selon les réactions suivantes :

\section{Réaction 1}

$$
\mathrm{CO}_{2}+3 \mathrm{H}_{2} \Leftrightarrow \mathrm{CH}_{3} \mathrm{OH}+\mathrm{H}_{2} \mathrm{O} \quad K_{1}^{*}
$$

\section{Réaction 2}

$$
\mathrm{CO}+\mathrm{H}_{2} \mathrm{O} \Leftrightarrow \mathrm{H}_{2}+\mathrm{CO}_{2} \quad K_{2}^{*}
$$

Les vitesses des réactions (13) et (14) sont données par Froment et al. [12]. 


\section{PROCÉDURE DE RÉSOLUTION DU MODĖLE MATHÉMATIQUE}

Le modèle mathématique du réacteur de synthèse de méthanol est basé sur deux types d'équations de conservation qui sont :

- type 1 : les équations 11 et 12 de la phase fluide ;

- type 2 : les équations de diffusion de 5 à 8 .

La procédure utilisée consiste à résoudre de façon itérative les deux types d'équations. Le système d'équation (type 2) fourni la distribution de la température et de la concentration molaire de chaque constituant à l'intérieur de la particule de catalyseur, qui est nécessaire à l'évaluation du facteur d'efficacité. Après quoi, les équations (type 1) sont intégrées pour déterminer les profils des concentrations et de température le long du réacteur.

\subsection{Intégration des équations de la phase fluide}

Les profils axiaux de concentration et température sont obtenus par intégration simultanée des équations 11 et 12 par la méthode de Runge-Kutta de quatrième ordre [13]. Le gradient de pression donné par la relation 3 est calculé séparément à la fin de chaque pas d'intégration $d z$.

\subsection{Intégration des équations de diffusion}

Le facteur d'efficacité est calculé le long du réacteur après intégration des équations de diffusion et à chaque pas d'intégration. Il permet la correction du modèle cinétique vu que ce dernier est obtenu sur une poudre fine de même type de catalyseur où les phénomènes de diffusion n'interviennent pas.

La méthode numérique d'intégration des équations de diffusion est la collocation orthogonale [14-19]. Son principe consiste à approximer les variables dépendantes par une interpolation polynomiale avec $N$ points appelés collocation. Le polynôme utilisé pour l'approximation des dérivées est celui de Lagrange. La discrétisation de l'équation différentielle permet de transformer les équations différentielles en un système d'équations algébriques qui est résolu avec la méthode de Broyden [13] donnant les profils de température et de concentration molaire pour chaque constituant à l'intérieur de la particule de catalyseur. Ceci permet d'évaluer les vitesses des deux réactions d'où le calcul du facteur d'efficacité pour chaque réaction par un schéma itératif.

\section{SIMULATION DU RÉACTEUR}

\subsection{Caractéristiques du réacteur}

Le réacteur de synthèse de méthanol d'Arzew est constitué de quatre lits catalytiques, adiabatiques et superposés. Entre chaque lit, il est prévu une injection de gaz de synthèse frais pour refroidir les réactifs. Les caractéristiques du réacteur et du catalyseur ainsi que la composition molaire de l'alimentation du réacteur prévue par le design et réelle sont tabulées ci-dessous.

\section{TABLEAU}

Caractéristiques du réacteur de synthèse de méthanol Characteristics of the methanol synthesis reactor

\begin{tabular}{l|c}
\hline Hauteur de chaque lit catalytique & $0,75 \mathrm{~m}$ \\
Diamètre du réacteur & $3,90 \mathrm{~m}$ \\
fraction de vide du lit catalytique & 0,38 \\
\hline
\end{tabular}

TABLEAU 2

Caractéristiques du catalyseur Gas composition of the catalyst

\begin{tabular}{l|c}
\hline Type du catalyseur & $\mathrm{ZnO} / \mathrm{CuO} / \mathrm{Al}_{2} \mathrm{O}_{3}$ \\
Densité du solide & $1770 \mathrm{~kg} / \mathrm{m}^{3}$-solide \\
Diamètre de la particule & $5,4 \mathrm{~mm}$ \\
Fraction du vide de la particule & 0,24 \\
\hline
\end{tabular}

TABLEAU 3

Composition molaire du gaz à l'entrée du réacteur Gas composition of the reactor at the inlet

\begin{tabular}{c|c|c}
\hline \multirow{2}{*}{ Constituants } & $\begin{array}{c}\text { Composition molaire } \\
\text { prévue } \\
\text { par le design }(\%)\end{array}$ & $\begin{array}{c}\text { Composition molaire } \\
\text { réelle du réacteur } \\
\text { d'Arzew (\%) }\end{array}$ \\
\hline $\mathrm{CO}$ & 7,50 & 6,51 \\
$\mathrm{CO}_{2}$ & 6,00 & 7,45 \\
$\mathrm{H}_{2}$ & 73,70 & 71,02 \\
$\mathrm{H}_{2} \mathrm{O}$ & 0,13 & 0,48 \\
$\mathrm{CH}_{3} \mathrm{OH}$ & 0,05 & 0,03 \\
$\mathrm{~N}_{2}$ & 3,26 & 3,46 \\
$\mathrm{CH}_{4}$ & 9,36 & 11,05 \\
\hline
\end{tabular}

TABLEAU 4

Conditions opératoires du réacteur Operating conditions of the reactor

\begin{tabular}{l|c} 
Pression de fonctionnement (atm) & 51 \\
Débit $(\mathrm{kmol} / \mathrm{hr})$ & 14220 \\
Débit du premier lit $(\mathrm{kmol} / \mathrm{hr})$ & 9960 \\
Débit total de refroidissement $(\mathrm{kmol} / \mathrm{hr})$ & 4260
\end{tabular}

La température à l'entrée de chaque lit catalytique est de $230{ }^{\circ} \mathrm{C}$ et la température du gaz de refroidissement intermédiaire est de $70{ }^{\circ} \mathrm{C}$.

Les propriétés thermodynamiques (facteur de compressibilité et les écarts enthalpiques) sont calculées par l'équation 
d'état thermodynamique de Peng Robinson [10, 11]. Les propriétés physico-chimiques du fluide sont calculées à l'aide de corrélations $[10,11]$.

\subsection{Résultats et discussion}

Les résultats de la simulation du réacteur de synthèse de méthanol sont montrés à travers les figures 1 à 9 . La composition molaire à l'entrée du réacteur est ici celle donnée par le design.
Le profil de température (fig. 1) suit une allure croissante, ceci est dû à la nature exothermique des réactions. Le domaine de variation de température diminue d'un lit à un autre. Ceci est expliqué en examinant les vitesses de réactions présentées par la figure 3. Ce profil de vitesse est décroissant lorsque ces lits ont la même température d'entrée et la même quantité de catalyseur.

L'évolution des concentrations molaires est donnée par la figure 2 ci-dessous. Le profil du $\mathrm{CO}_{2}$ diminue avant de se

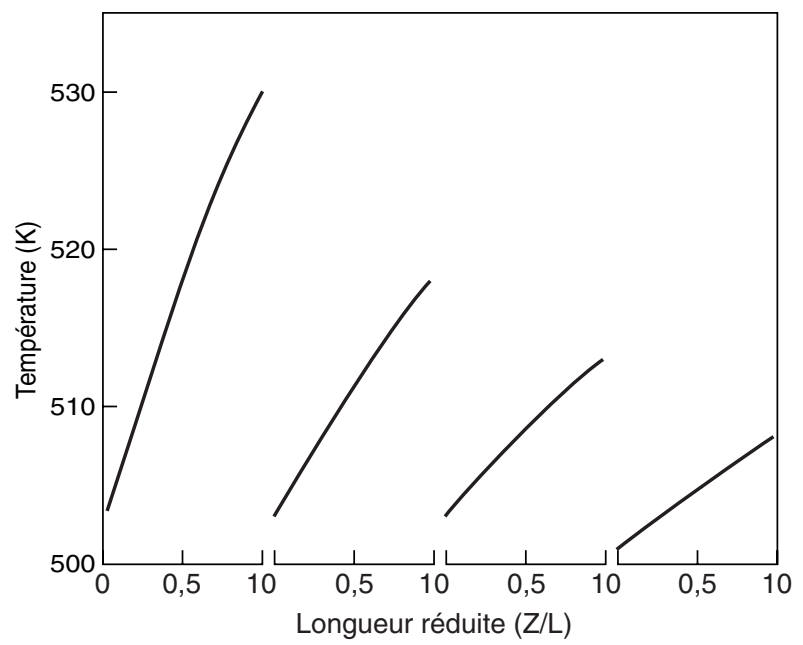

Figure 1

Profil de température : température longueur réduite pour les quatre lits catalytique.

Temperature profile: temperature-reduced length for the four catalytic beds.

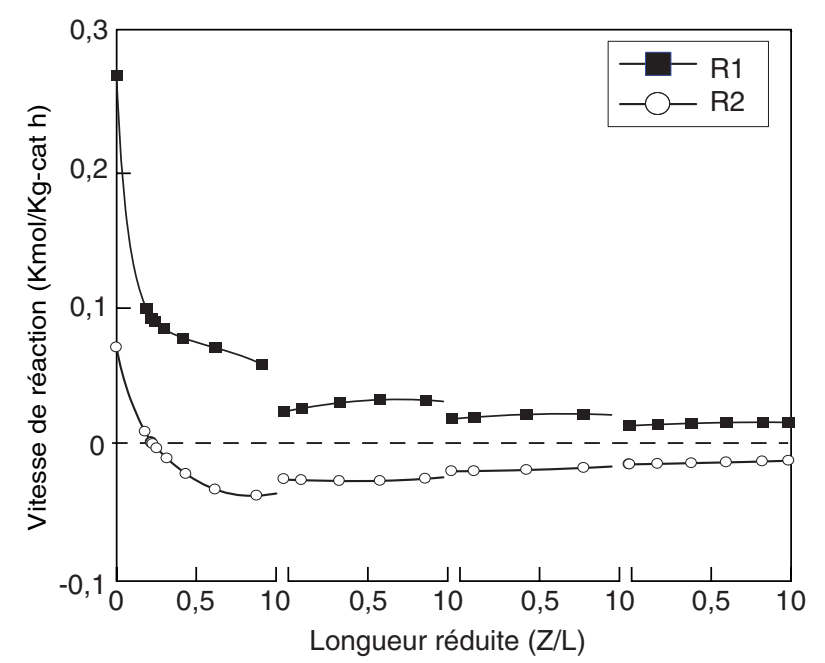

Figure 3

Profil de vitesse de réaction : vitesse de réaction-longueur réduite pour les quatre lits catalytique $(\mathrm{R} 1$ = réaction 1 ; $\mathrm{R} 2$ = réaction 2).

Reaction rate profile: reaction rate-reduced length for the four catalytic beds.

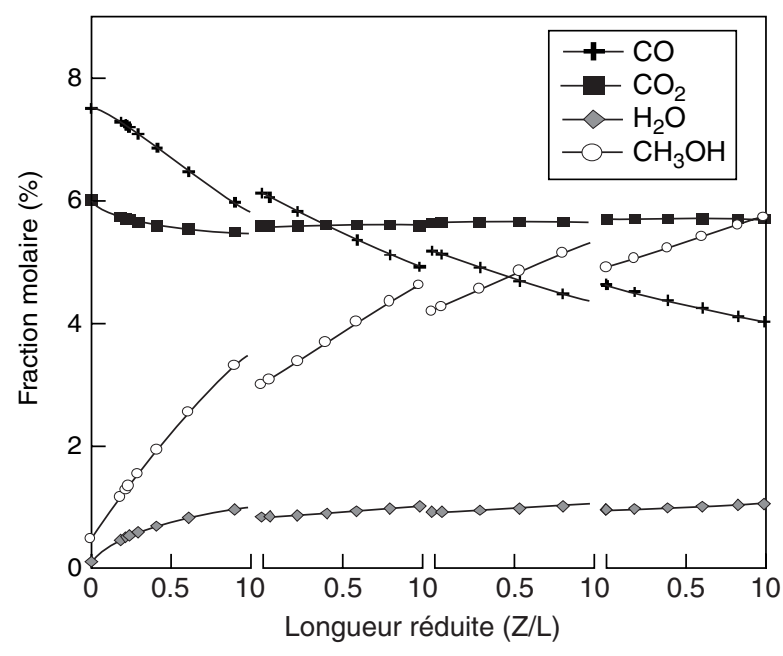

Figure 2

Profil de concentration : concentration longueur réduite pour les quatre lits catalytique.

Concentration profile: concentration-reduced length for the four catalytic beds.

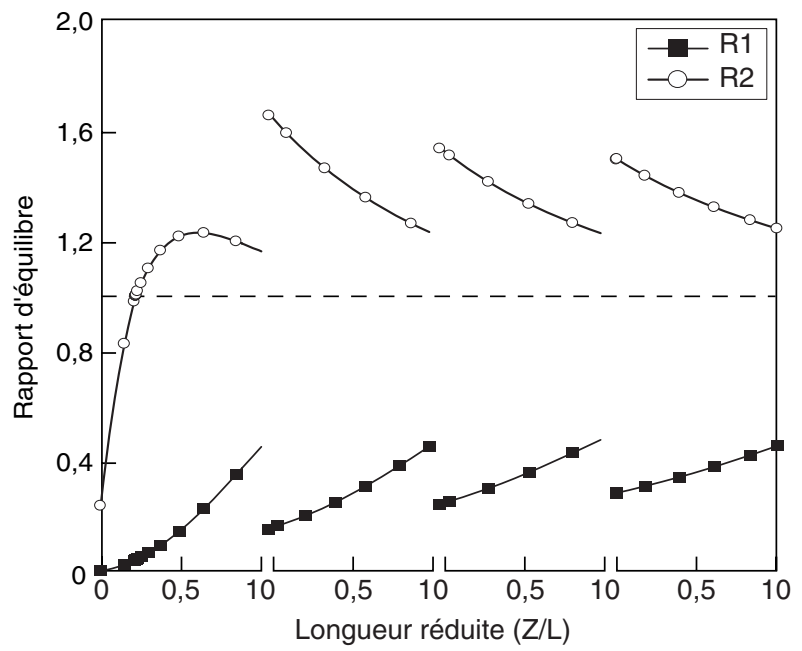

Figure 4

Profil de rapport d'équilibre de réaction : rapport d'équilibrelongueur réduite pour les quatre lits catalytique $(\mathrm{R} 1=$ réaction $1 ; \mathrm{R} 2=$ réaction 2 ).

Ratio of equilibrium reaction profile: ratio of equilibriumreduced length for the four catalytic beds. 
stabiliser à partir de $25 \%$ de la longueur du $1^{\text {er }}$ lit. Ceci s'explique par le fait que le $\mathrm{CO}_{2}$ est consommé par la réaction de synthèse de méthanol et en même temps il est produit par la réaction 2. Dans la figure 3, on voit que l'équilibre de la réaction est inversé. Au début, elle se déroule dans le sens de la consommation du $\mathrm{CO}_{2}$ et avec le changement de température et de composition molaire du système réactionnel, elle évolue dans le sens de la production du $\mathrm{CO}_{2}$.
Ce comportement est dû à la réversibilité de cette réaction qui est quantifiée par le rapport d'équilibre. Ce dernier quantifie l'approche d'une réaction chimique réversible à son équilibre thermodynamique et est présenté dans la figure 4.

La vitesse de réaction est établie pour un constituant de coefficient stœchiométrique positif, et le signe négatif ne peut provenir que dans le cas ou ce rapport d'équilibre dépasse la valeur de un (1). Le changement de signe de la vitesse de

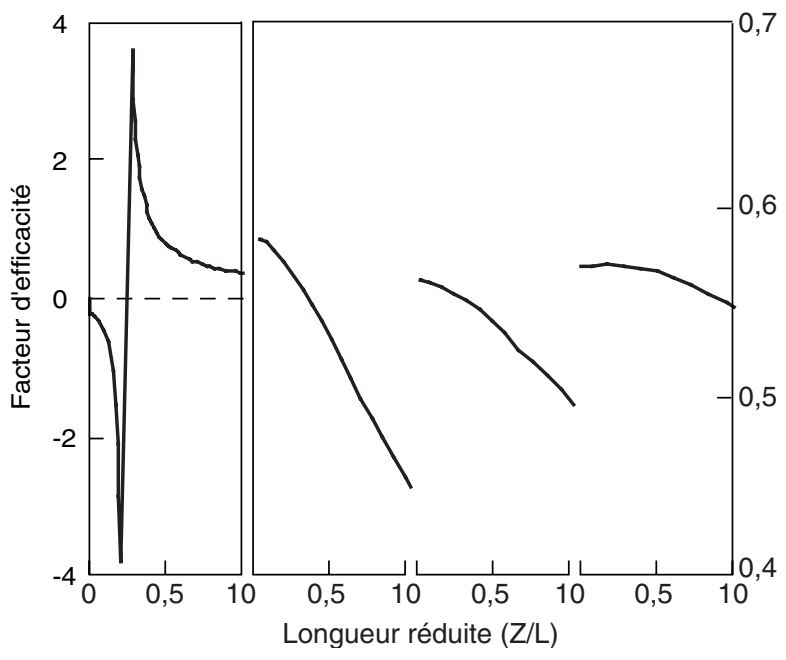

Figure 5

Profil du facteur d'efficacité de la réaction $2: \eta_{2}$-longueur réduite pour les quatre lits catalytique.

Effectiveness factor profile for reaction $R 2: \eta_{2}$-reduced length for the four catalytic beds.

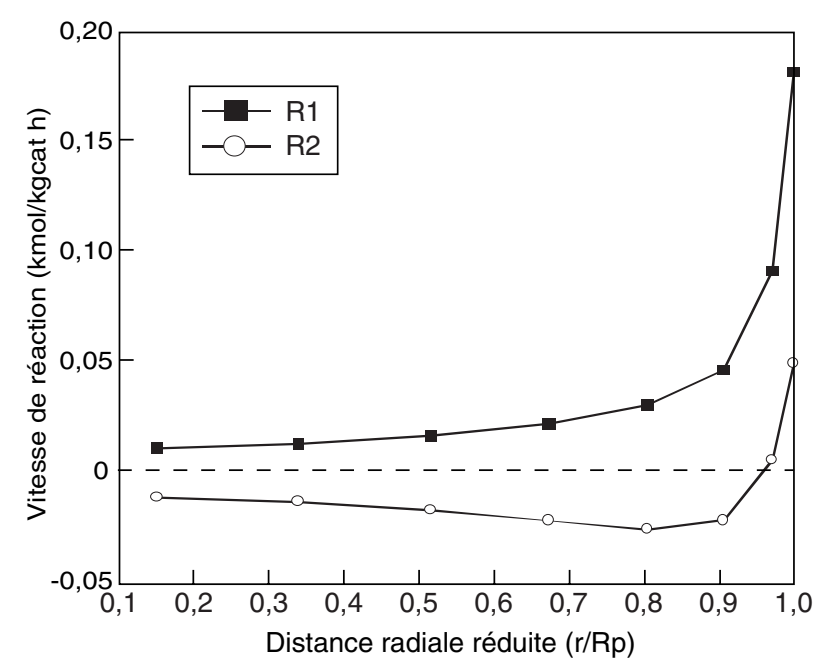

Figure 7

Profil de vitesse : vitesse-distance radiale réduite à l'intérieur de la particule à $\mathrm{Z} / \mathrm{L}=0,06 \mathrm{du} 1^{\mathrm{er}}$ lit $(\mathrm{R} 1=$ réaction 1 ; $\mathrm{R} 2$ = réaction 2 )

Reaction rate profile: reaction rate-intraparticule radial reduced distance, $Z / L=0.06$ for the first bed.

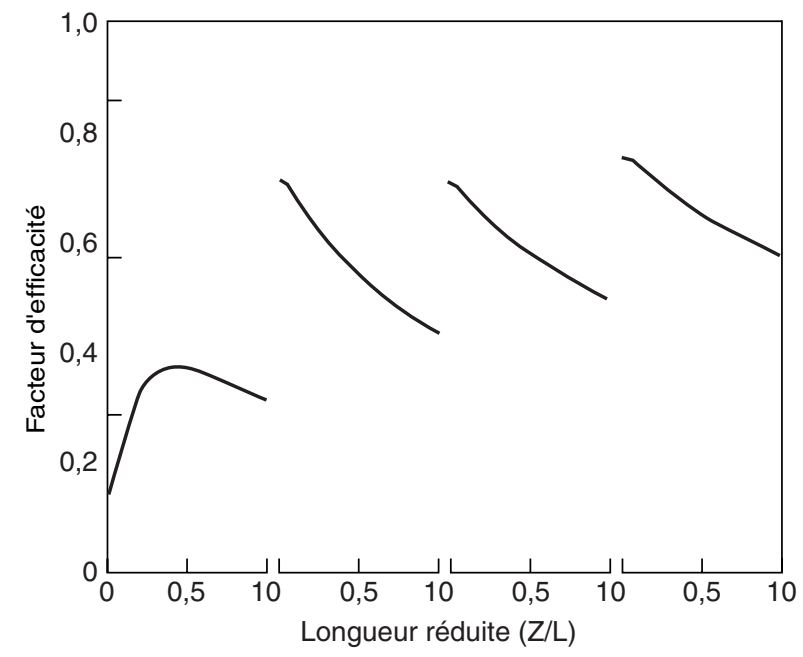

Figure 6

Profil du facteur d'efficacité de la réaction $1: \eta_{1}$-longueur réduite pour les quatre lits catalytique.

Effectiveness factor profile for reaction R1: $\eta_{1}$-reduced length for the four catalytic beds.

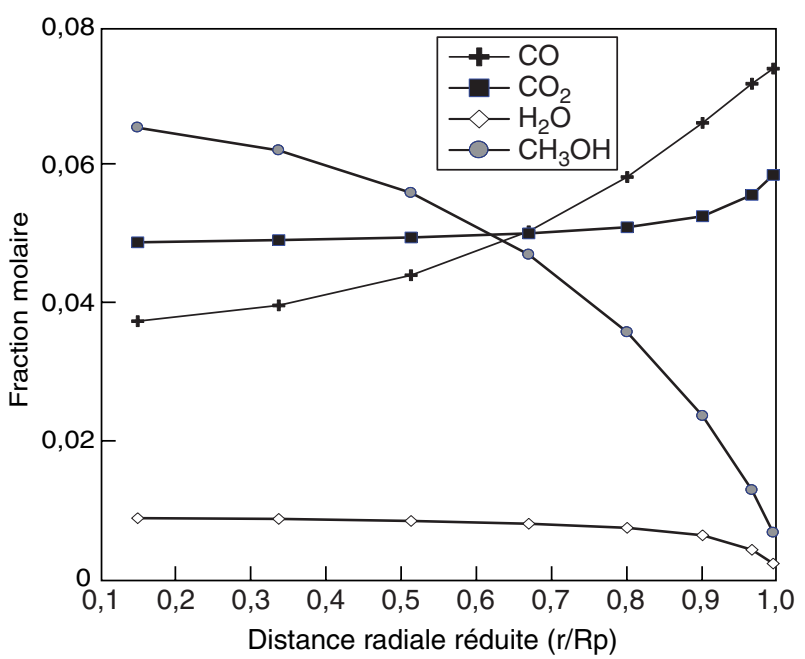

Figure 8

Profil de concentration : concentration-distance radiale réduite à l'intérieur de la particule et $\mathrm{Z} / \mathrm{L}=0,06 \mathrm{du} 1^{\mathrm{er}}$ lit.

Concentration profile: concentration-intraparticule radial reduced distance, $Z / L=0.06$ for the first bed. 
réaction 2 est expliqué par le rapport d'équilibre présenté dans la figure 4. A l'entrée du réacteur, ce rapport est inférieur à un (1) $R_{2}>0$, puis devient égal à un (1) à l'équilibre $\left(R_{2}=0\right)$. Suite à la présence de la réaction de synthèse de méthanol réaction 1 se déroulant en parallèle avec la réaction 2, l'équilibre de cette dernière est déplacé. Dans les nouvelles conditions de température et de concentration, le rapport d'équilibre de la réaction 2 devient supérieur à un (1), ce qui donne des valeurs de vitesses négatives.

Ce comportement de la réaction 2 a une incidence directe sur le facteur d'efficacité de la réaction 2 porté sur la figure 5. À l'entrée du premier lit, il est négatif et proche de zéro, puis il continue vers des valeurs négatives $(-\infty)$. À $25 \%$ de la longueur du lit, il passe de $(-\infty)$ à $(+\infty)$, avant d'atteindre une valeur de l'ordre de 0.5 à la sortie du premier lit. L'explication de ce comportement est déduite de l'expression mathématique équation (10). Le facteur d'efficacité est un rapport de deux vitesses, celle qui se déroule à l'intérieur de la particule à celle calculée aux conditions de la phase fluide.

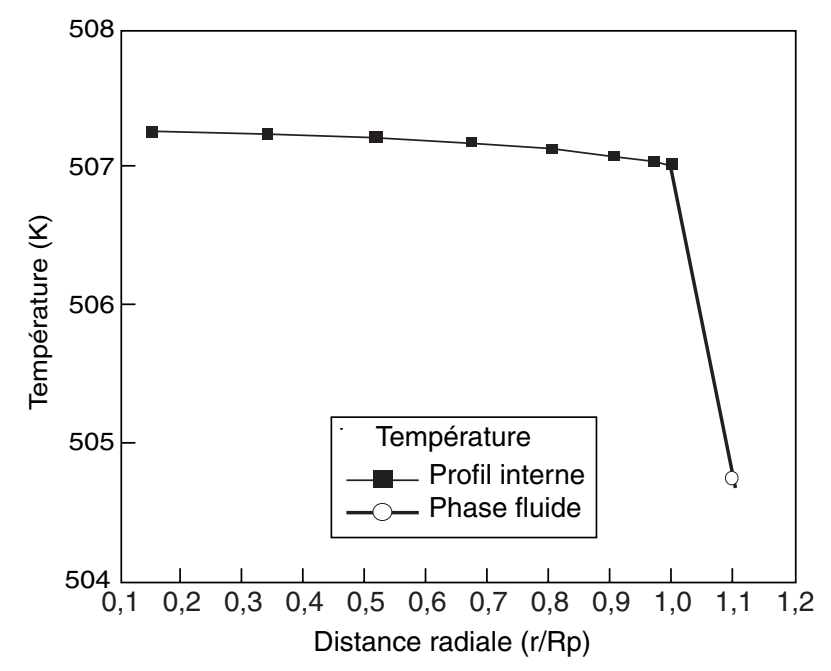

Figure 9

Profil de température : température-distance radiale à l'intérieur de la particule et $\mathrm{Z} / \mathrm{L}=0,06 \mathrm{du} 1^{\mathrm{er}}$ lit.

Temperature profile: temperature-intraparticule radial reduced distance, $Z / L=0.06$ for the first bed.

Le facteur d'efficacité de la réaction de synthèse de méthanol, porté sur la figure 6 , a des valeurs faibles à l'entrée du premier lit, avec une évolution croissante jusqu'à $50 \%$ de la longueur du lit où il atteint la valeur de 0.4. Pour les autres lits, le facteur d'efficacité a une forme décroissante et un ordre de grandeur compris entre 0.6 et 0.4 .
À partir de la figure 7, on peut remarquer que la réaction 2 se déroule dans les deux sens. À l'intérieur de la particule elle est négative et proche de la surface de la particule, elle est positive. Ce comportement du facteur d'efficacité est expliqué par le suivi du profil du rapport d'équilibre. La vitesse globale de la réaction 2 à l'intérieur de la particule se déroule dans le sens de la consommation du $\mathrm{CO}_{2}$ avec des valeurs négatives et dans le sens opposé valeurs positives au sein du fluide. Le facteur d'efficacité est le rapport de ces deux vitesses.

La figure 8 dresse les profils des concentrations molaires en fonction de la distance radiale réduite à l'intérieur de la particule de catalyseur pour le premier lit catalytique.

Enfin, le gradient de température le plus élevé est obtenu au niveau du film enveloppant la particule du catalyseur figure 9 . Ce résultat est en accord avec la littérature $[3,4]$ où il est suggéré de considérer que la particule du catalyseur est isotherme et que la résistance au transfert thermique se situe uniquement dans le film mais pour le transfert de matière, c'est le contraire, c'est-à-dire qu'on peut négliger la résistance dans le film et tenir compte uniquement du gradient à l'intérieur du solide.

La simulation du réacteur de synthèse de méthanol a contribué énormément à la compréhension du système réactionnel et en particulier la réaction du gaz à l'eau (Water Gas Shift) et ceci grâce à l'accès à un grand nombre d'informations tels que les profils de vitesse et le rapport d'équilibre au sein de la phase fluide et à l'intérieur de la particule de catalyseur.

\subsection{Comparaison avec les données du design}

Les résultats de simulation sont dans un premier temps comparés avec ceux du design.

À partir du tableau 5, on voit que la production du méthanol simulée est plus importante que celle donnée par le design à cause de deux facteurs dont le premier est la valeur très faible de la tortuosité prise, constante et égale à 2 pour la simulation du cas design, ce qui réduit la résistance au transfert de matière à l'intérieur de la particule de catalyseur et favorise donc la conversion en méthanol. Le deuxième facteur pouvant expliquer l'écart, réside dans le fait que notre modèle ne prend pas en compte la désactivation du catalyseur, surtout qu'il est connu que :

- le catalyseur du premier lit se désactive rapidement tandis que les lits subséquents se désactivent moins rapidement [20];

- la production d'un réacteur industriel est établie par le constructeur sur la base d'une moyenne en tenant compte de la durée de vie du catalyseur.

À partir de là, on peut considérer que la comparaison des résultats de simulation à ceux du design est acceptable d'autant plus que la composition à la sortie du premier lit figure 2 est pratiquement du même ordre de grandeur que celle donnée par le design. 
TABLEAU 5

Comparaison des résultats de la composition des produits à la sortie du lit $\mathrm{n}^{\circ} 4$ donnée par le design avec ceux de la simulation Comparison of the products molar composition at the exit bed number 4 between design and simulation

\begin{tabular}{|c|c|c|c|}
\hline \multirow[b]{2}{*}{ Constituants } & \multicolumn{3}{|c|}{ Composition molaire (\%) } \\
\hline & $\begin{array}{c}\text { entrée lit no }{ }^{\circ} 1 \\
\text { design }\end{array}$ & $\begin{array}{c}\text { sortie lit } n^{\circ} 4 \\
\text { design }\end{array}$ & $\begin{array}{c}\text { sortie lit } n^{\circ} 4 \\
\text { simulation }\end{array}$ \\
\hline $\mathrm{CO}$ & 7,50 & 6,01 & 4,03 \\
\hline $\mathrm{CO}_{2}$ & 6,00 & 5,30 & 5,70 \\
\hline $\mathrm{H}_{2}$ & 73,70 & 71,53 & 70,04 \\
\hline $\mathrm{H}_{2} \mathrm{O}$ & 0,13 & 1,20 & 1,05 \\
\hline $\mathrm{CH}_{3} \mathrm{OH}$ & 0,05 & 3,06 & 5,72 \\
\hline $\mathrm{N}_{2}$ & 3,26 & 3,35 & 3,53 \\
\hline $\mathrm{CH}_{4}$ & 9,36 & 9,55 & 9,93 \\
\hline
\end{tabular}

TABLEAU 6

Comparaison des températures d'entrée et de sortie obtenues par le calcul avec ceux du design Comparison of the inlet and exit bed temperatures between design and simulation

\begin{tabular}{c|c|c|c|c}
\hline \multirow{2}{*}{ Lit } & \multicolumn{2}{|c|}{ Température en $\left({ }^{\circ} \mathrm{C}\right)$ à l'entrée de chaque lit } & \multicolumn{2}{|c}{ Température en $\left({ }^{\circ} \mathrm{C}\right)$ à la sortie de chaque lit } \\
\cline { 2 - 4 } & design & simulation & design & Simulation \\
\hline 1 & 503 & 503 & $<543$ & 530 \\
2 & 503 & 503 & $<543$ & 518 \\
3 & 503 & 503 & $<543$ & 513 \\
4 & 503 & 501 & $<543$ & 508 \\
\hline
\end{tabular}

TABLEAU 7

Comparaison des résultats de simulation de la composition des produits à la sortie du lit $\mathrm{n}^{\circ} 4$ avec ceux du réacteur industriel sur une moyenne de fonctionnement d'une année et après changement de catalyseur

Comparison of the products molar composition at the exit bed number 4 between industrial reactor and simulation

\begin{tabular}{|c|c|c|c|}
\hline \multirow[b]{2}{*}{ Constituants } & \multicolumn{3}{|c|}{ Composition molaire (\%) } \\
\hline & $\begin{array}{l}\text { entrée lit } \mathrm{n}^{\circ} 1 \\
\text { réacteur industriel }\end{array}$ & $\begin{array}{l}\text { sortie lit } n^{\circ} 4 \\
\text { réacteur industriel }\end{array}$ & $\begin{array}{c}\text { sortie lit } \mathrm{n}^{\circ} 4 \\
\text { simulation }\end{array}$ \\
\hline $\mathrm{CO}$ & 6,51 & 5,76 & 5,16 \\
\hline $\mathrm{CO}_{2}$ & 7,45 & 6,75 & 6,85 \\
\hline $\mathrm{H}_{2}$ & 71,02 & 68,25 & 68,19 \\
\hline $\mathrm{H}_{2} \mathrm{O}$ & 0,48 & 1,47 & 1,26 \\
\hline $\mathrm{CH}_{3} \mathrm{OH}$ & 0,03 & 2,98 & 3,15 \\
\hline $\mathrm{N}_{2}$ & 3,46 & 3,55 & 3,76 \\
\hline $\mathrm{CH}_{4}$ & 11,05 & 11,24 & 11,63 \\
\hline
\end{tabular}




\subsection{Comparaison avec les données réelles du fonctionnement du réacteur d'Arzew}

Les résultats de simulation du réacteur sont comparés avec ceux du fonctionnement réel du réacteur. Il y a lieu de noter que la composition molaire à l'entrée du réacteur est différente de celle donnée par le design (tableau 3).

On peut remarquer que les résultats de simulation obtenus sont très proches de ceux du réacteur réel. Il est à préciser toutefois que ces résultats ont été obtenus avec une tortuosité égale à 8 après l'avoir fait varier de 2 à 10 .

\subsection{Influence du $\mathrm{CO}_{2}$ sur la synthèse du méthanol}

La source de carbone dans le méthanol provient du $\mathrm{CO}$ et du $\mathrm{CO}_{2}$. Il s'ensuit que la quantité du carbone converti en méthanol dépend du rapport $\mathrm{P}_{\mathrm{CO} 2} / \mathrm{P}_{\mathrm{CO}}$. L'étude de l'influence de la composition du $\mathrm{CO}_{2}$ et du $\mathrm{CO}$ sur la synthèse est réalisée avec deux approches.

\subsubsection{Concentration constante en carbone}

La démarche consiste à faire varier la composition molaire du $\mathrm{CO}_{2}$ et du $\mathrm{CO}$ sans affecter la composition des autres constituants. La teneur totale en carbone du gaz entrant au réacteur de synthèse du méthanol est la somme de la teneur du $\mathrm{CO}_{2}$ plus celle du $\mathrm{CO}$, qui est de 13,5\% molaire à l'entrée du réacteur dans le cas design.

Les résultats de simulation pour le premier lit catalytique sont présentés par les figures 10 et 11 qui montrent que la fraction molaire du méthanol passe par un maximum qui correspond à $\mathrm{P}_{\mathrm{CO} 2} / \mathrm{P}_{\mathrm{CO}}=0,3$. Ce résultat n'est pas attendu du fait que le terme cinétique de la vitesse de réaction de synthèse de méthanol est fonction uniquement de la pression partielle de l'hydrogène et du $\mathrm{CO}_{2}$ mais il s'explique par la faible vitesse de réaction pour un taux important de $\mathrm{CO}_{2}$.

\subsubsection{Apport externe en $\mathrm{CO}_{2}$}

L'alimentation est enrichie en $\mathrm{CO}_{2}$ provenant d'une source externe. Les résultats sont résumés sur la figure 12. Elle montre qu'il n'est pas intéressant d'ajouter du $\mathrm{CO}_{2}$ à l'alimentation du réacteur, parce que le rapport actuel $\left(\mathrm{P}_{\mathrm{CO} 2} / \mathrm{P}_{\mathrm{CO}}=0.8\right)$ du gaz de synthèse alimentant le réacteur industriel d'Arzew se trouve loin du rapport de production maximale de méthanol.

\subsubsection{Proposition d'amélioration de la production en méthanol du réacteur $d^{\prime} A r z e w$}

Pour augmenter la production de méthanol à partir du réacteur industriel d'Arzew et valoriser ainsi toute source de $\mathrm{CO}_{2}$, il faut que le $\mathrm{CO}_{2}$ entre dans la production du gaz de synthèse. Ainsi on peut obtenir un gaz riche en carbone. Le $\mathrm{CO}_{2}$ peut être transformé en $\mathrm{CO}$ par la réaction de gaz à l'eau (Water Gas Shift Reaction) comme le montre (fig. 13) le modèle cinétique du reformage à la vapeur du méthane [9].

Cette solution sera intéressante dans la mesure où un gaz de synthèse peut être produit avec un rapport $\mathrm{CO}_{2} / \mathrm{CO}$ optimal comme le montre la figure 14. La production de

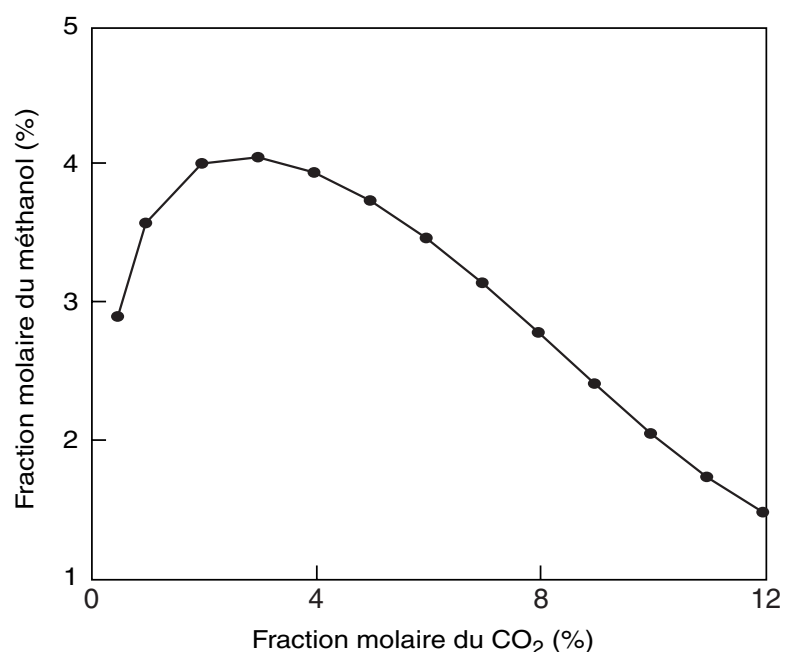

Figure 10

Profil de production du méthanol : fraction molaire-concentration du $\mathrm{CO}_{2}$ à l'entrée du réacteur.

Methanol production profile: molar fraction-concentration of $\mathrm{CO}_{2}$ at the inlet of the reactor

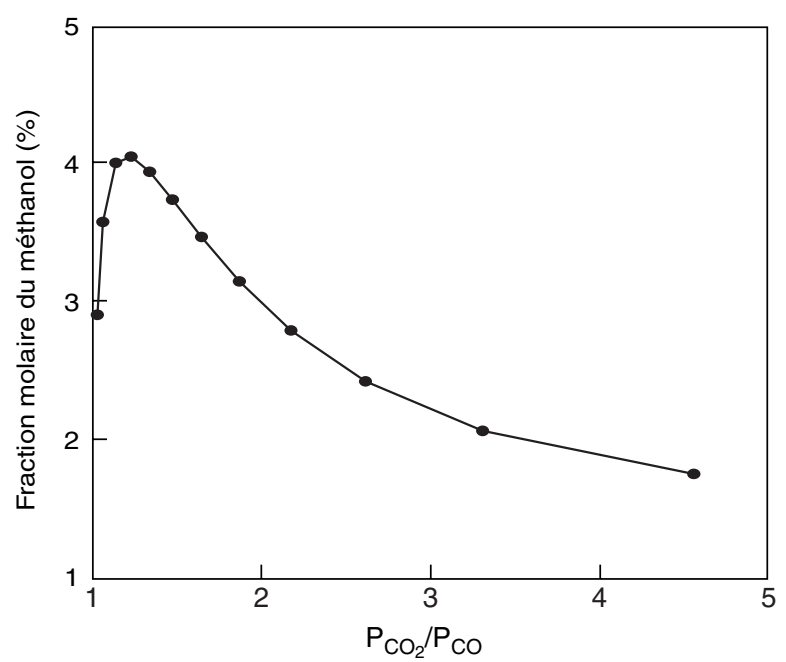

Figure 11

Profil de production du méthanol : fraction molaire-rapport $\mathrm{P}_{\mathrm{CO} 2} / \mathrm{P}_{\mathrm{CO}}$ à l'entrée du réacteur.

Methanol production profile: molar fraction-ratio $P_{\mathrm{CO} 2} / P_{\mathrm{CO}}$ at the inlet of the reactor 
méthanol dépend du rapport $\mathrm{P}_{\mathrm{CO} 2} / \mathrm{P}_{\mathrm{CO}}$ et de la teneur totale en carbone présent sous forme de $\mathrm{CO}_{2}$ et de $\mathrm{CO}$. La production est maximale pour le même rapport $\mathrm{P}_{\mathrm{CO} 2} / \mathrm{P}_{\mathrm{CO}}$ qui est de l'ordre de 0.3. Cette proposition est réalisable vu le développement qu'a connu le procédé de reformage à la vapeur où on peut produire un gaz de synthèse riche en carbone [21]. L'intérêt de cette proposition se situe surtout dans l'abandon de l'ancienne idée fausse sur l'appoint direct de $\mathrm{CO}_{2}$ au niveau du réacteur de synthèse de méthanol d'Arzew et d'orienter l'étude dans le sens de cette nouvelle proposition.

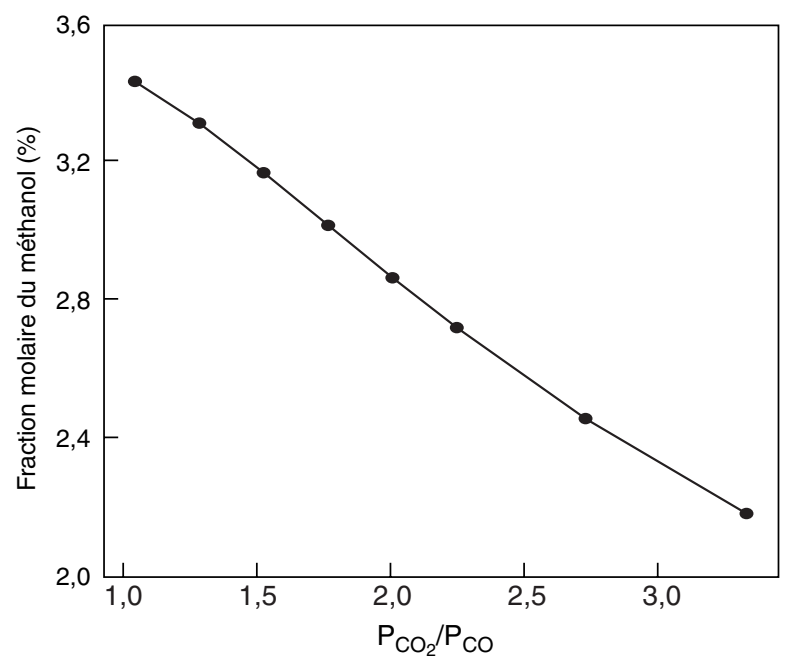

Figure 12

Profil de production du méthanol : fraction molaire-rapport $\mathrm{P}_{\mathrm{CO} 2} / \mathrm{P}_{\mathrm{CO}}$.

Methanol production profile: molar fraction-ratio $P_{\mathrm{CO}_{2}} / P_{\mathrm{CO}}$

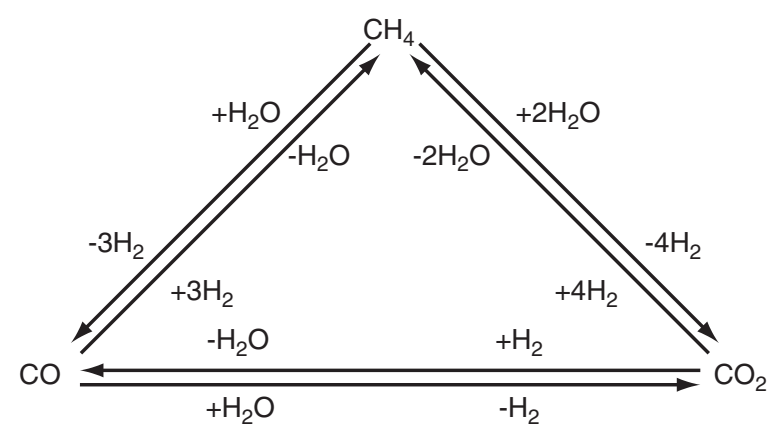

Figure 13

Modèle de réactions du reformage à la vapeur du méthane. Model of steam reforming reactions of methane.

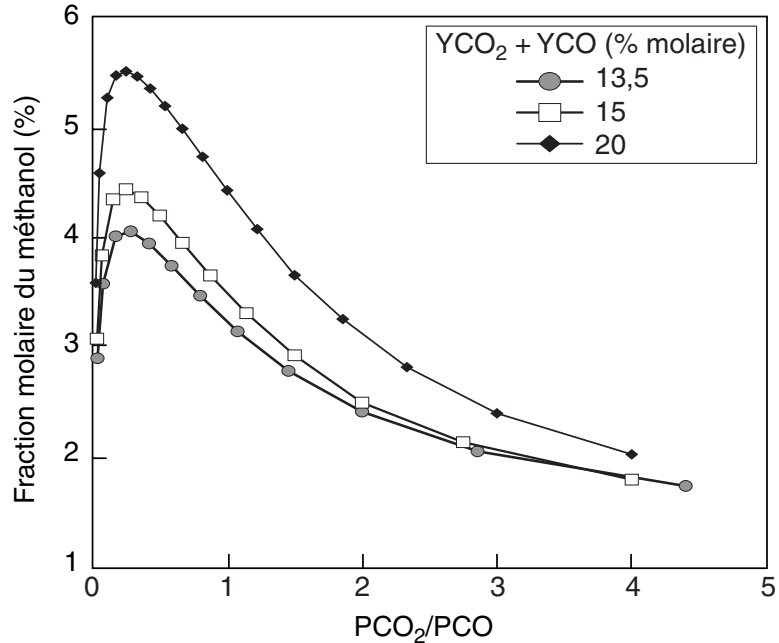

Figure 14

Profil de production du méthanol : fraction molaire-rapport $\mathrm{P}_{\mathrm{CO} 2} / \mathrm{P}_{\mathrm{CO}}$ pour différentes quantités de $\left(\mathrm{CO}_{2}+\mathrm{CO}\right)$.

Methanol production profile : molar fraction-ratio $P_{\mathrm{CO} 2} / P_{\mathrm{CO}}$ for different amount of $\left(\mathrm{CO}_{2}+\mathrm{CO}\right)$.

\section{CONCLUSION}

Dans ce travail nous avons montré l'importance de la modélisation et de la simulation pour la compréhension du comportement du réacteur de synthèse de méthanol d'Arzew mettant en jeu des réactions chimiques parallèles et réversibles.

Le modèle utilisé dans ce travail permet la détermination des vitesses de réaction dans la phase fluide et à l'intérieur de la particule ainsi que les profils des concentrations molaires et de température à l'intérieur de la particule qui sont inaccessibles avec l'utilisation des moyens de mesure. Ce travail a également permis l'investigation du facteur d'efficacité pour des réactions parallèles et réversibles avec un modèle cinétique complexe. Enfin, il a permis la détermination du rapport optimal $\left(\mathrm{P}_{\mathrm{CO} 2} / \mathrm{P}_{\mathrm{CO}}=0.3\right)$ du gaz de synthèse du Complexe Pétrochimique d'Arzew qui maximise la production en méthanol du réacteur d'Arzew. Enfin, les résultats de simulation obtenus sont proches des données industrielles du réacteur d'Arzew.

\section{REFERENCES}

1 Bird R.B., Steward W.E. and Lightfoot E.N. (1960) Transport phenomena, Wiley.

2 Slattery J.C. (1980) Momentum, energy, and mass transfer in continua. 2nd edition, Mc Graw Hill.

3 Froment G.F. and Bischoff K.B. (1990) Chemical reactor analysis and design. 2nd edition, Wiley, 393-506. 
4 Froment G.F. and Hofmann H.P. (1987) Design of fixed-bed gas-solid catalytic reactor, 373-440, in Carberry, J.J., Varma, A., Chemical reactor and reactor engineering, Marcel Dekker.

5 Villermaux J. (1993), Génie de la réaction chimique conception et fonctionnement des réacteurs, TEC \& DOC Lavoisier, $2^{\mathrm{e}}$ édition, 348-358.

6 Villermaux J. (1993), Génie de la réaction chimique conception et fonctionnement des réacteurs, TEC \& DOC Lavoisier, 2 édition, 397-400.

7 Westerterp K.R., Van Swaaij W.P.M. and Beenackers A.A.C.M. (1984), Chemical reactor design and operation, Wiley, 622-624.

8 Froment G.F. and Bischoff K.B. (1990), Chemical reactor analysis and design, 2nd Edition, Wiley, 130-131.

9 Doraiswamy L.K. and Sharma M.M. (1984), Heterogeneous reactions: Analysis, examples and reactor design, Wiley, $52-54$.

10 Reid R.C., Prausnitz J.M. and Sherwood T.K. (1977) The properties of gases and liquids, Mc Graw Hill, 3rd Edition.

11 Reid R.C., Prausnitz J.M. and Poling B.E. (1987) The properties of gases and liquids, McGraw Hill, 4th Edition.

12 Froment G.F. and Vanden Bussche K.M. (1996), A steadystate model for methanol synthesis and water gas shift reaction on a commercial $\mathrm{Cu} / \mathrm{ZnO} / \mathrm{Al}_{2} \mathrm{O}_{3}$ catalyst, J. Catal. 161, 1-10.

13 Numerical recipes software (V2.01) Méthode de Rung-Kutta de cinquième ordre et la Méthode de Broyden.

14 Villadsen J. and Michelsen M.L. (1979), Solution of differential equation models by polynomial approximation. PrenticeHall.
15 Villadsen J. and Michelsen M.L. (1987) Numerical methods in reaction engineering, $\mathrm{p}(1-62)$, cité dans J.J. Carberry, A. Varma, Chemical reactor and reactor engineering, Marcel Dekker.

16 Kjaer J. (1972) Computer methods in differential equations, Topsoe Haldor, Denmark, 91.

17 Rice R.G. and Do D.D. (1995) Applied mathematics and modeling for chemical engineers, Wiley, 268-314.

18 Michelsen M.L. and Villadsen J. (1972) A convenient computation procedure for collocation constants, Chem. Eng. J., 6468.

19 Christiansen L.J. and Jarven Jorgen E. (1985) Transport restriction in catalyst particles with several chemical reaction, Paper presented at the NATO conference on chemical reactor design and technology, Canada.

20 Islam K.A. and Earl W.B. (1990) Deactivation of ICI low temperature methanol catalyst in an industrial reactor, Can. J. Chem. Eng., 68, 702-704.

21 Bogild Hansen J. and Joensen F. (1991) High conversion of synthesis gas into oxygenates. Cité dans Natural gas conversion, A. Holmen et al., 457-467.

22 Velardi S., Barressi A.A. and Antonello A. (2002) Methanol synthesis in a forced unsteady-state reactor network, Chem. Eng. Sci., 57, 2995-3004.

23 Shahrokhi M. and Baghmisheh G.R. (2005) Modelling, simulation and control of a methanol synthesis fixed-bed reactor, Chem. Eng. Sci., 60, 4275-4286.

Manuscrit définitif reçu en avril 2006

Copyright (c) 2006 Institut français du pétrole

Permission to make digital or hard copies of part or all of this work for personal or classroom use is granted without fee provided that copies are not made or distributed for profit or commercial advantage and that copies bear this notice and the full citation on the first page. Copyrights for components of this work owned by others than IFP must be honored. Abstracting with credit is permitted. To copy otherwise, to republish, to post on servers, or to redistribute to lists, requires prior specific permission and/or a fee: Request permission from Documentation, Institut français du pétrole, fax. +33147527078 , or revueogst@ifp.fr. 